\title{
Use of constrained synthetic amino acids in $\beta$-helix proteins for conformational control
}

\section{David Zanuy ${ }^{1}$, Ana I. Jiménez ${ }^{2}$, Carlos Cativiela ${ }^{2}$, Ruth Nussinov ${ }^{3,4^{*}}$ and Carlos Alemán ${ }^{1 ; *}$}

\footnotetext{
${ }^{\text {I}}$ Departament d'Enginyeria Química, ETSEIB, Universitat Politècnica de Catalunya, Diagonal 647, 08028 Barcelona (Spain)

${ }^{2}$ Departamento de Química Orgánica, ICMA, Universidad de Zaragoza-CSIC, 50009 Zaragoza (Spain)

${ }^{3}$ Basic Research Program, SAIC-Frederick, Inc. Center for Cancer Research Nanobiology Program, NCI, Frederick, MD 21702 (USA)

${ }^{4}$ Department of Human Genetics, Sackler Medical School, Tel Aviv University, Tel Aviv 69978 (Israel)
}

Corresponding authors: carlos.aleman@upc.edu, ruthn@ncifcrf.gov

\begin{tabular}{clc}
\hline \# & Page & Description \\
\hline Figure S1 & S2 & Graphical representation of krr1 $1_{131-165}$ crystalline structure \\
Figure S2 & S3 & fluctuations and Spatio-temporal evolution of backbone RMSD in MD series I \\
Figure S3 & S4 & fluctuations and Spatio-temporal evolution of backbone RMSD in MD series II \\
Figure S4 & S5 & Snapshots corresponding to the simulation of wild type model in MD series I \\
Figure S5 & S6 & Snapshot corresponding to the simulation of model I in MD series I \\
Figure S6 & S7 & Amino acids sequence of krr1 131-165 \\
Table S1 & S8 & All minima found through systematic conformational search at HF/ 6-31G(d) level \\
Table S2 & S9 & Comparison of the energy differences computed at HF/ 6-31G(d) and B3LYP/6-311++G(d,p) level \\
Table S3 & S10 & Conformational features of the Gly-Xxx-Gly loop. \\
Table S4 & S11 & \\
\hline
\end{tabular}



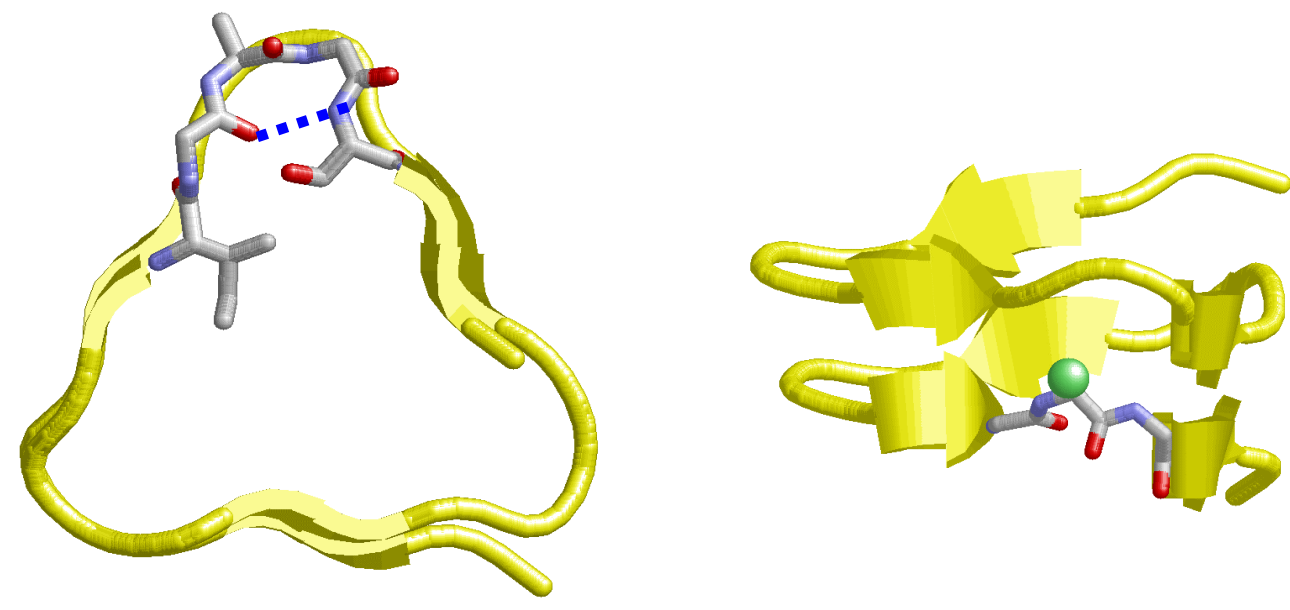

Figure S1. Crystal structure of the left-handed $\beta$-sheet structure of the system investigated in the present study, residues 131-165 of Galactoside acetyltransferase from E. coli (PDB code krr1): top view (left) and front view (right). Atoms are represented as solid ribbons in yellow. In the top view, the residues around the mutated Ala160 are explicitly depicted, the $\mathrm{C}_{10}$ hydrogen bond ( $\beta$-turn) between the $\mathrm{C}=\mathrm{O}$ of Gly159 and the $\mathrm{N}-\mathrm{H}$ of Ser162 being specifically indicated. In the front view, all the atoms with exception of Ala160 are omitted for clarity. A green ball represents the methyl side group of Ala160. 
(a)

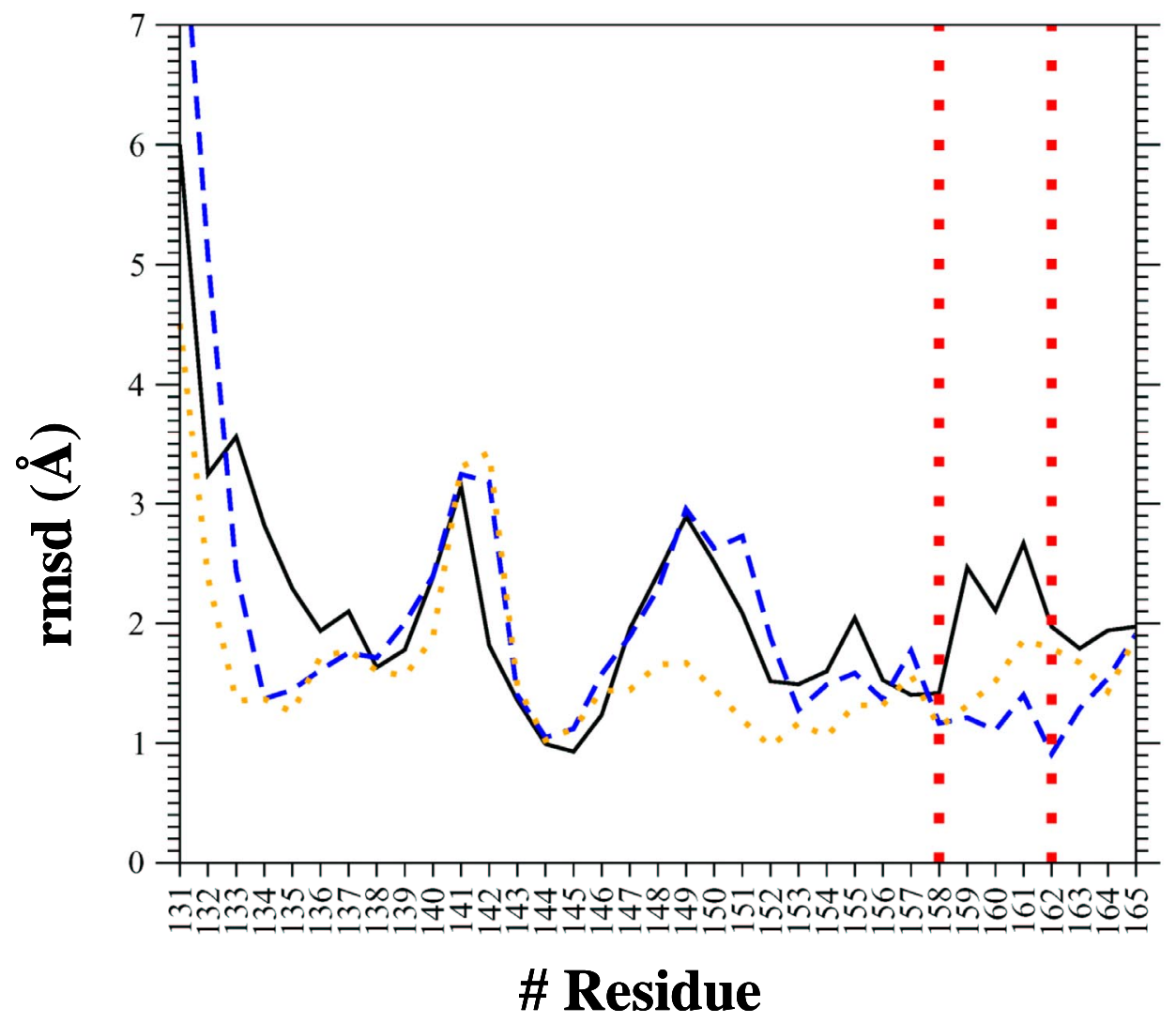

(b)

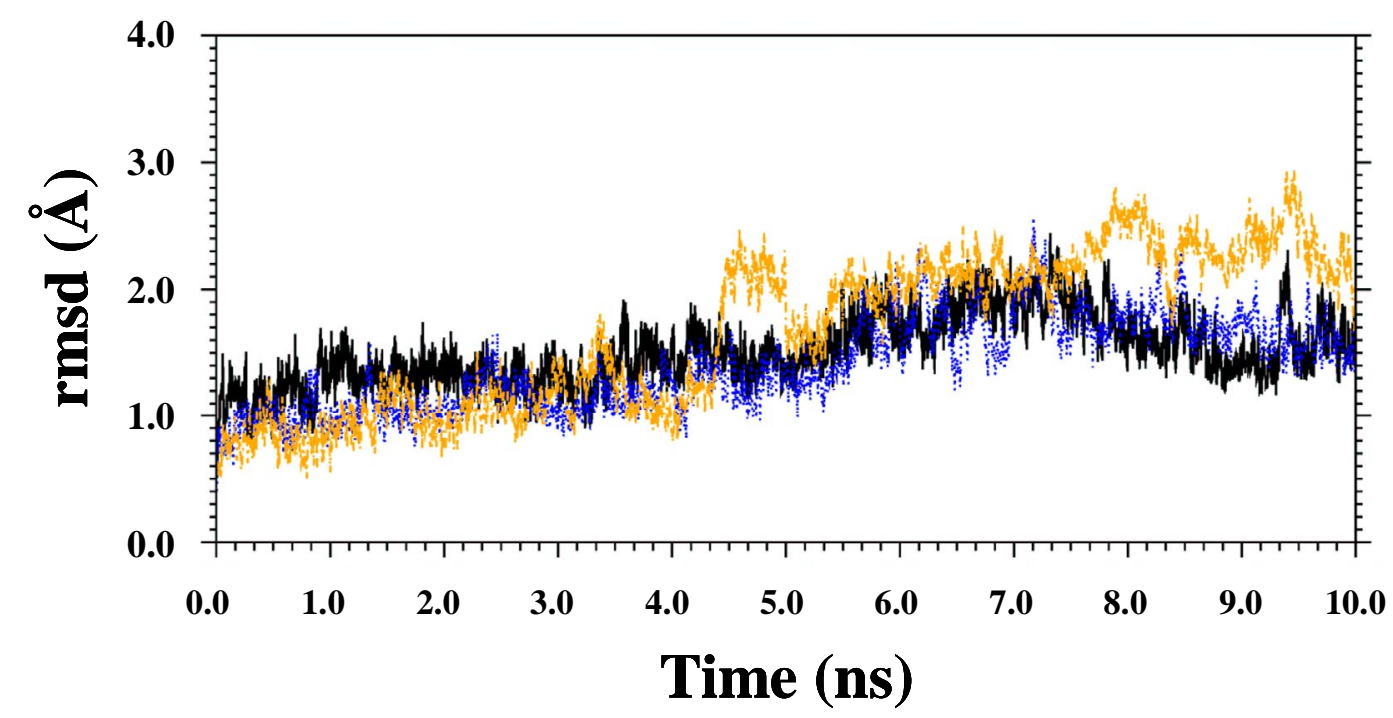

Figure S2. Second series of simulations: (a) Average rmsd for the 35 residues of the simulated systems and (b) temporal evolution of the rmsd calculated without considering the residues at the $\mathrm{N}_{\mathrm{t}}$-extreme: wild type (black), model I (orange) and model II (blue). 
(a)

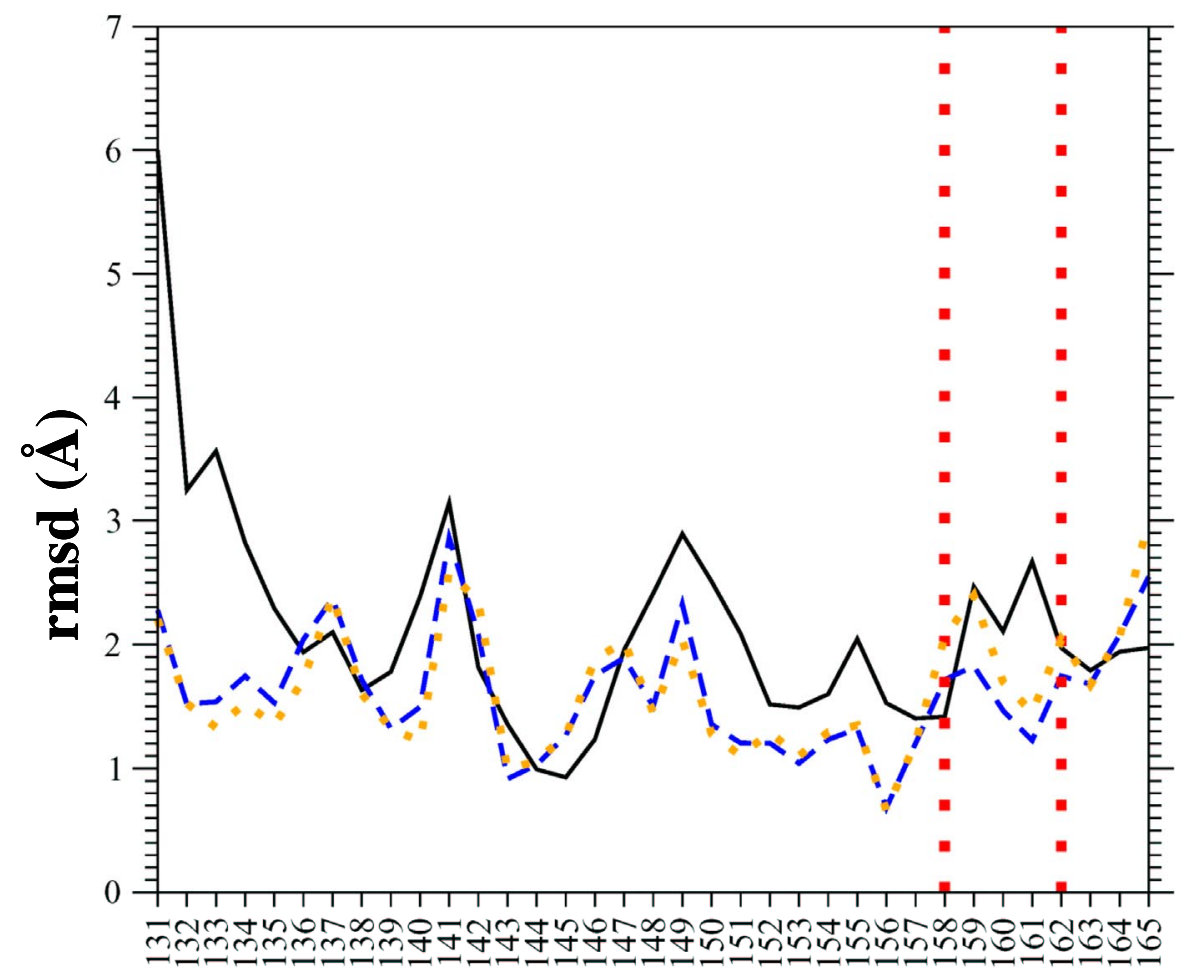

\# Residue

(b)

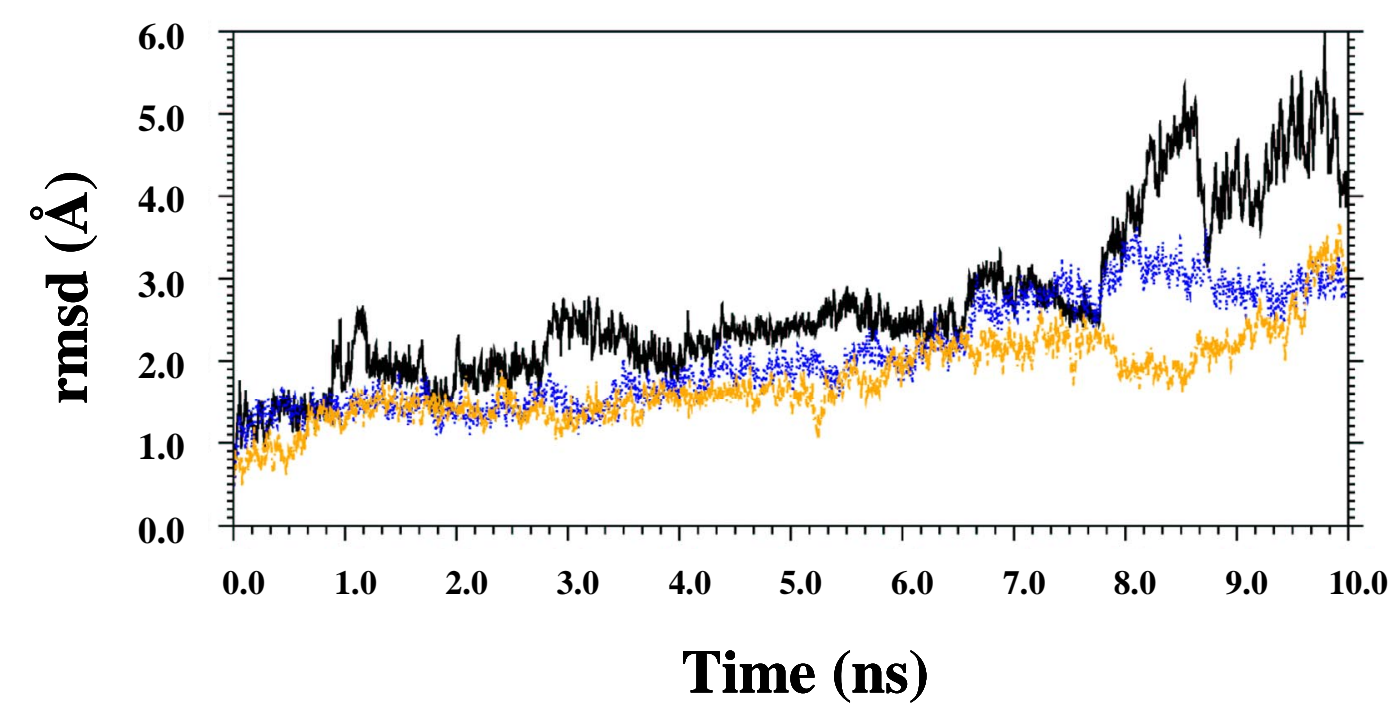

Figure S3. Third series of simulations: (a) Average rmsd for the 35 residues of the simulated systems and (b) temporal evolution of the rmsd calculated without considering the residues at the $\mathrm{N}_{\mathrm{t}}$-extreme: wild type (black), model I (orange) and model II (blue). 

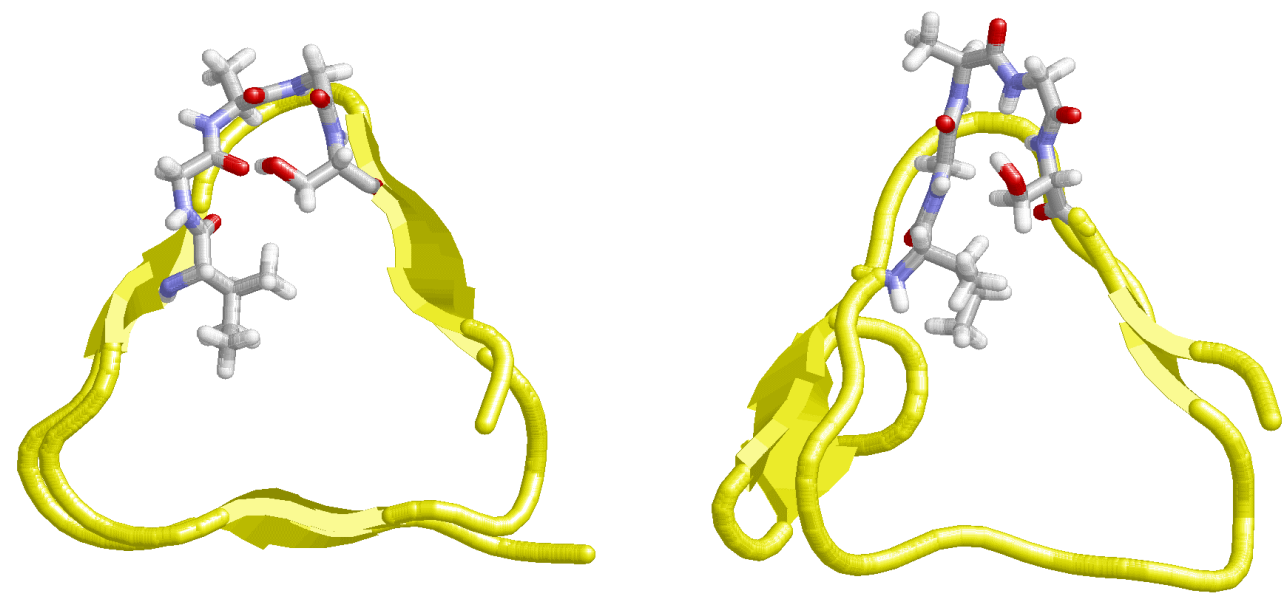

Figure S4. For the wild-type system: snapshots recorded after 0.25 (left) and $10 \mathrm{~ns}$ (right) of MD simulation. Atoms are represented as solid ribbons in yellow. The residues around Ala160 are explicitly depicted. As can be seen, the $\mathrm{C}_{10}$ intraturn hydrogen bond found in the crystal structure of krr1 (see Figure S1) disappears after a few steps of MD simulation. 


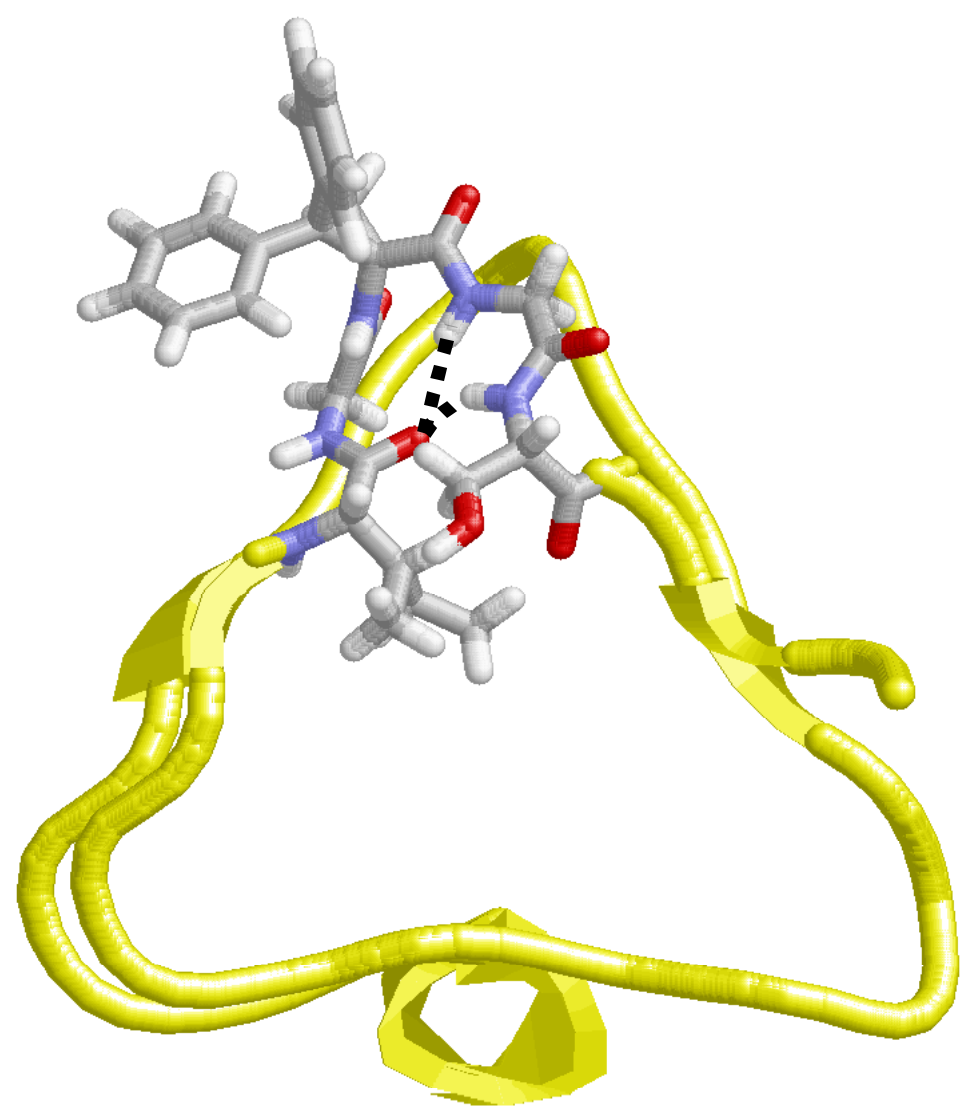

Figure S5. Intraturn $\mathrm{C}_{10}$ and $\mathrm{C}_{13}$ hydrogen bonds formed between the $\mathrm{C}=\mathrm{O}$ of Ile 158 and the N-H of both Gly161 and Ser162 during the MD simulation of model I. Hydrogen bonds are depicted using a black dashed line. 


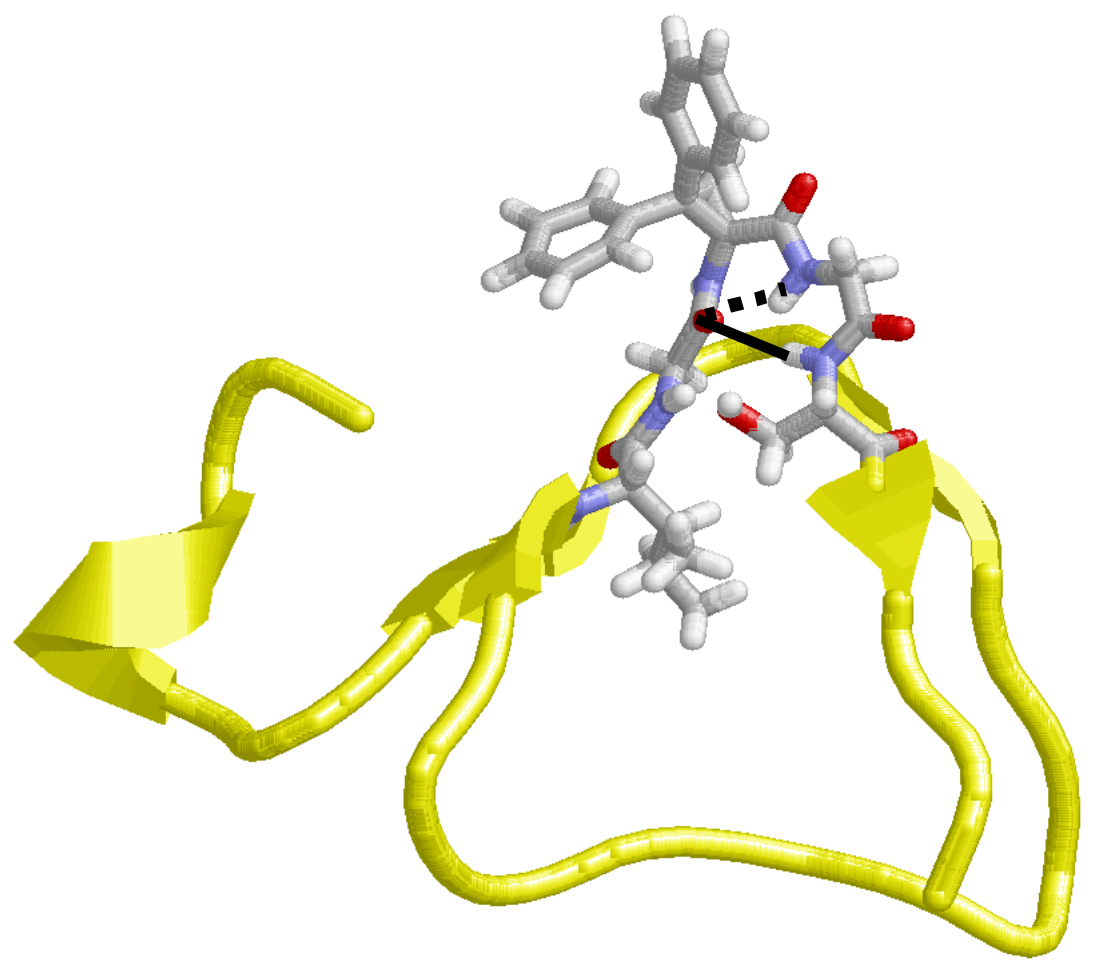

Figure S6. Intraturn $\mathrm{C}_{7}$ and $\mathrm{C}_{10}$ hydrogen bonds formed between the $\mathrm{C}=\mathrm{O}$ of Gly 159 and the N-H of both Gly161 and Ser162 during the MD simulation of model II. Hydrogen bonds are depicted using a black dashed line. 
Table S1. Sequence of E. coli Galactoside acetyltransferase, residues 131-165.

\section{PITIGNNVWIGSHVVINPGVTIGDNSVIGAGSIVT}


Table S2. Minimum energy conformations for the Ac-Gly-L-c ${ }_{3}$ Dip-Gly-NHMe tripeptide obtained at the ab initio HF/6-31G(d) level.

\begin{tabular}{|c|c|c|c|c|c|c|c|}
\hline \multicolumn{2}{|c|}{ Gly } & \multicolumn{2}{|c|}{ c3Dip } & \multicolumn{2}{|c|}{ Gly } & & \multirow[b]{2}{*}{$\Delta \mathrm{E}(\mathrm{kcal} / \mathrm{mol})$} \\
\hline$\varphi$ & $\psi$ & $\varphi$ & $\psi$ & $\varphi$ & $\psi$ & & \\
\hline-178 & -179.8 & -76.8 & -25.7 & 76.7 & -172.6 & -176.6 & 3.29 \\
\hline 76.3 & 25.9 & -73.7 & -26 & 75.3 & 90.3 & -63.9 & 4.92 \\
\hline-73.6 & -9.8 & -69.6 & -23.8 & 78.2 & -84.5 & 63.1 & 3.41 \\
\hline 178.6 & -174.5 & -76.2 & -27.5 & 76.5 & -84.4 & 76.6 & 3.69 \\
\hline 89.5 & -66.7 & -68.5 & -26 & 71.7 & -132.5 & 162.5 & 1.27 \\
\hline-87.6 & 57.1 & -74.7 & -24.1 & 73.4 & -163 & -1769 & 3.94 \\
\hline 76.5 & 24.3 & -71.3 & -32.2 & 76.1 & -84.9 & 79 & 4.93 \\
\hline-70.9 & -17.5 & -72.6 & -20.9 & 77.9 & 87.9 & -60.3 & 2.42 \\
\hline-130.6 & 17.9 & -61.9 & 126.9 & 75.6 & 98.2 & -10 & 3.50 \\
\hline-79.1 & 167.1 & -69.7 & 127.7 & 75.1 & -124.4 & 18.8 & 4.73 \\
\hline-179.3 & 176.2 & -71.8 & 134.5 & 75.3 & 176.8 & 175.3 & 2.46 \\
\hline-178.7 & 177.4 & -71.3 & 127.8 & 74 & -124.7 & 19.6 & 3.79 \\
\hline 123.5 & -22.6 & -66.2 & 135 & 77.1 & 176.5 & 174.9 & 5.67 \\
\hline-133.1 & 19.5 & -75.2 & 122.8 & 74 & -118.6 & 18.1 & 5.51 \\
\hline-75.5 & 162.8 & -60.2 & 129.8 & 76.1 & 96.6 & -9.1 & 2.94 \\
\hline-175.8 & 166.7 & 76.4 & -39.6 & 79.2 & -177.4 & 173.3 & 4.65 \\
\hline-126.4 & 27.8 & 75.8 & -45.1 & 77 & -85.7 & 75.4 & 4.64 \\
\hline-174.5 & 168.3 & 76.5 & -41.7 & 78.4 & 86.6 & -61.7 & 3.06 \\
\hline-175.4 & -168.2 & 76.3 & -45.5 & 77.9 & -87 & 69.9 & 3.62 \\
\hline 84.7 & -65.3 & 77.3 & -36.6 & 79 & -171.6 & 174.5 & 3.27 \\
\hline-142.5 & -1322 & 71 & -58.9 & 73.4 & -72 & 159.5 & 2.39 \\
\hline 84.4 & -65 & 76.3 & -44.9 & 76.8 & -86.7 & 68.4 & 1.25 \\
\hline-126.9 & 25.7 & 76 & -43.7 & 77 & 86.4 & -59.3 & 4.53 \\
\hline 177.7 & -173 & 64.2 & 178 & 76.8 & 171.5 & -178 & 6.88 \\
\hline 124.8 & -26.7 & 64.1 & -174.1 & 75.1 & 88.2 & -72.2 & 8.31 \\
\hline-79.1 & 167.1 & -70 & 127.7 & 75.1 & -124.4 & 18.8 & 6.52 \\
\hline 178.4 & -174.4 & 63.7 & -174.6 & 74.9 & 88.4 & -72.2 & 6.38 \\
\hline 177.9 & -173.7 & 63.3 & -176.3 & 75.7 & -85.1 & 57.9 & 5.98 \\
\hline 142.9 & -27.9 & 64.8 & 179.3 & 76.8 & 172 & -178.2 & 9.16 \\
\hline-156.6 & 50.3 & 61.8 & -179.8 & 74.6 & 171.6 & 179.6 & 7.28 \\
\hline 140.7 & -26.4 & 63.3 & -174.5 & 75.5 & -85 & 55.2 & 7.82 \\
\hline-151.5 & 47 & 61.8 & -171.9 & 73.1 & 89.1 & -63.4 & 7.24 \\
\hline-70.6 & -17.5 & -63.3 & -22.7 & 78.2 & -90.8 & 5.3 & 1.50 \\
\hline-99.8 & -80.3 & -68.3 & -25.2 & 77.1 & -112.4 & 19.4 & 8.65 \\
\hline-137 & 20.2 & -75.4 & 130.3 & 75.8 & 177.3 & 175.5 & 5.52 \\
\hline-172.5 & 166.6 & 78 & -41.9 & 79.3 & -113.1 & 12 & 5.12 \\
\hline-87.3 & 66.8 & 69.3 & -58.8 & 71.8 & -77.4 & 152.5 & 0.91 \\
\hline-82.3 & 83.3 & 72.9 & -46.5 & 76.9 & -80 & 92.1 & 0.98 \\
\hline-149.5 & 45 & 54.7 & -149.4 & 69.8 & -87.3 & 10.8 & 6.26 \\
\hline 176.9 & -173.9 & 52.2 & -147 & 66.7 & -90.3 & 12.7 & 6.33 \\
\hline-151.8 & 47.1 & 60 & -174.4 & 73.1 & -86 & 48.6 & 6.52 \\
\hline-99.8 & -80.3 & -68.3 & -25.2 & 77.1 & -112.4 & 19.8 & 3.77 \\
\hline 76.7 & 23.7 & -69.7 & -27.3 & 75.8 & -99.3 & 14.4 & 3.52 \\
\hline 85.8 & 11.8 & -69.7 & 125.3 & 76.3 & -121.8 & 17.1 & 5.24 \\
\hline 74.3 & 22.8 & 74.1 & -43.8 & 77.5 & -123.1 & 10.2 & 3.32 \\
\hline 141.2 & -25.6 & 57.7 & -138.6 & 65.9 & -93.2 & 12.8 & 7.57 \\
\hline-64.2 & -38 & -91 & 125.3 & 73.9 & 104.5 & -30.6 & 4.62 \\
\hline-152 & 48.3 & 62 & -177.3 & 74.5 & 104 & -7.8 & 8.50 \\
\hline 61.1 & -132.8 & -69.7 & -21.3 & 76.8 & 88.2 & -56.1 & 1.50 \\
\hline 178.7 & -175.1 & -78.5 & -22.8 & 76.4 & 89.1 & -64 & 3.34 \\
\hline 89.5 & -66.6 & -68.5 & -26 & 71.7 & -132.5 & 162.5 & 1.27 \\
\hline 62.9 & -127 & -62.4 & -23.9 & 77.1 & -90.2 & 4.7 & 0.00 \\
\hline 82.3 & -78.1 & -61.3 & 133.3 & 69.1 & -128.9 & 18.7 & 2.90 \\
\hline-177.6 & 173.4 & -60.4 & 130.4 & 75.4 & 97.6 & -9.8 & 2.46 \\
\hline 82.5 & -78.3 & -61.3 & 141.6 & 69.8 & 177.7 & 175.6 & 3.28 \\
\hline 86.7 & -67.7 & -75.6 & 91.2 & 67.9 & 66.8 & -159.5 & 1.96 \\
\hline-85.9 & 68.5 & 74.6 & -42.3 & 77.5 & 86.5 & -59 & 2.12 \\
\hline 84.5 & -63.1 & 67.4 & 177.7 & 79.4 & 172.3 & -177.1 & 7.09 \\
\hline 84 & -61.8 & 65.4 & -175.9 & 77.8 & -84.4 & 54.3 & 5.48 \\
\hline-80.9 & 80.9 & 63.7 & -173.4 & 74.5 & 89.2 & -57.6 & 5.01 \\
\hline 85.3 & -63.8 & 77 & -41.6 & 78.2 & 86.5 & -59.6 & 0.79 \\
\hline 85.3 & 11.5 & -61.8 & 128 & 76.9 & 96.1 & -8.6 & 2.72 \\
\hline 81.4 & -80.5 & -55.3 & 133.5 & 70.1 & 93.8 & -7.9 & 0.80 \\
\hline-126.9 & 25.7 & 76 & -43.7 & 77 & 86.8 & -59.3 & 4.53 \\
\hline
\end{tabular}


Table S3. Conformational details of those energy minima obtained at HF/6-31G(d) level that presented a relative energy respect the absolute minimum smaller or equal to 3 $\mathrm{kcal} / \mathrm{mol}$. Main chain dihedral angles and the hydrogen bonds geometry (if present; see text) are included. Relative energies computed at B3LYP/6-311++G(d,p) level are also incorporated for comparison.

\begin{tabular}{|c|c|c|c|c|c|c|c|c|}
\hline \multicolumn{2}{|c|}{ Gly } & \multicolumn{2}{|c|}{$\mathbf{c}_{3}$ Dip } & \multicolumn{2}{|c|}{ Gly } & \multirow[b]{2}{*}{$\begin{array}{c}\text { Hydrogen } \\
\text { bonds } \\
\text { geometries }\end{array}$} & \multirow[b]{2}{*}{$H F^{\mathbf{a}}$} & \multirow[b]{2}{*}{$D F T^{\mathrm{a}}$} \\
\hline$\varphi$ & $\psi$ & $\varphi$ & $\psi$ & $\varphi$ & $\psi$ & & & \\
\hline 62.9 & -127.0 & -62.4 & -23.9 & -90.2 & 4.7 & $\mathrm{C}_{10}-\mathrm{C}_{10}$ & 0.00 & 0.00 \\
\hline 85.3 & -63.8 & 77.0 & -41.6 & 86.5 & -59.6 & $\mathrm{C}_{7}-\mathrm{C}_{7}-\mathrm{C}_{7}$ & 0.79 & 0.56 \\
\hline 81.4 & -80.5 & -55.3 & 133.5 & 93.8 & -7.9 & $\mathrm{C}_{7}-\mathrm{C}_{10}$ & 0.80 & 1.06 \\
\hline-87.3 & 66.8 & 69.3 & -58.8 & -77.4 & 152.5 & $\mathrm{C}_{11}-\mathrm{C}_{7}$ & 0.91 & 1.06 \\
\hline-82.3 & 83.3 & 72.9 & -46.5 & -80.0 & 92.1 & $\mathrm{C}_{7}-\mathrm{C}_{7}-\mathrm{C}_{7}$ & 0.98 & 0.38 \\
\hline 84.4 & -65.0 & 76.3 & -44.9 & -86.7 & 68.4 & $\mathrm{C}_{7}-\mathrm{C}_{7}-\mathrm{C}_{7}$ & 1.25 & 0.38 \\
\hline 89.5 & -66.6 & -68.5 & -26.0 & 132.5 & 162.5 & $\mathrm{C}_{11}$ & 1.27 & 1.62 \\
\hline 89.5 & -66.7 & -68.5 & -26.0 & -132.5 & 162.5 & $\mathrm{C}_{7}-\mathrm{C}_{11}$ & 1.27 & 1.62 \\
\hline-70.6 & -17.5 & -63.3 & -22.7 & -90.8 & 5.3 & none & 1.50 & 1.00 \\
\hline 61.1 & -132.8 & -69.7 & -21.3 & 88.2 & -56.1 & $\mathrm{C}_{10}-\mathrm{C}_{7}$ & 1.50 & 1.56 \\
\hline 86.7 & -67.7 & -75.6 & 91.2 & 66.8 & -15.5 & $\mathrm{C}_{7}-\mathrm{C}_{7}-\mathrm{C}_{10}$ & 1.96 & 3.56 \\
\hline-85.9 & 68.5 & 74.6 & -42.3 & 86.5 & -59.0 & $\mathrm{C}_{7}-\mathrm{C}_{7}-\mathrm{C}_{7}$ & 2.12 & 0.83 \\
\hline-142.5 & -132.2 & 71.0 & -58.9 & -72.0 & 159.5 & $\mathrm{C}_{11}-\mathrm{C}_{7}$ & 2.39 & 2.25 \\
\hline-70.9 & -17.5 & -72.6 & -20.9 & 87.9 & -60.3 & none & 2.42 & 2.19 \\
\hline-179.3 & 176.2 & -71.8 & 134.5 & 176.8 & 175.3 & $\mathrm{C}_{10}$ & 2.46 & 1.94 \\
\hline 82.3 & -78.1 & -61.3 & 133.3 & -128.9 & 18.7 & $\mathrm{C}_{7}$ & 2.90 & 3.75 \\
\hline-75.5 & 162.8 & -60.2 & 129.8 & 96.6 & -9.1 & $\mathrm{C}_{10}$ & 2.94 & 2.94 \\
\hline
\end{tabular}

${ }^{a}$ Energy differences computed respect to the absolute minimum in $\mathrm{kcal} / \mathrm{mol}$ 
Table S4. Backbone dihedral angles for the Gly159-Xxx160-Gly161 sequence used to build the three systems simulated using molecular dynamics.

\begin{tabular}{ccccc}
\hline System & Gly159 & Xxx160 & Gly161 & Source \\
\hline Wild-type & $\varphi, \psi=-66.7^{\circ}, 157.5^{\circ}$ & $\varphi, \psi=-57.6^{\circ}, 140.0^{\circ}$ & $\varphi, \psi=79.7^{\circ}, 0.6^{\circ}$ & PDB krr1 (X-ray data) \\
Mutant I & $\varphi, \psi=62.9^{\circ},-127.0^{\circ}$ & $\varphi, \psi=-62.4^{\circ},-23.9^{\circ}$ & $\varphi, \psi=-90.2^{\circ}, 4.7^{\circ}$ & Global minimum $^{\mathrm{a}}$ \\
Mutant II & $\varphi, \psi=-75.5^{\circ}, 162.8^{\circ}$ & $\varphi, \psi=-60.2^{\circ}, 129.8^{\circ}$ & $\varphi, \psi=96.6^{\circ},-9.1^{\circ}$ & Local minimum $^{\mathrm{a}}$ \\
\hline
\end{tabular}

${ }^{\mathrm{a}}$ Minima characterized for the tripeptide Ac-Gly-L-c 3 Dip-Gly-NHMe. 